\title{
Isotopic effects in impurity-vacancy complexes in diamond
}

\author{
E. A. Ekimov ${ }^{1}$, V. S. Krivobok ${ }^{2}$, S. G. Lyapin ${ }^{1}$, P. S. Sherin ${ }^{3,4}$, V. A. Gavva ${ }^{5}$, M. V. Kondrin ${ }^{1}$ \\ ${ }^{1}$ Institute for High Pressure Physics RAS, 142190 Troitsk, Moscow, Russia \\ ${ }^{2}$ Lebedev Physical Institute RAS, 117942, Leninsky pr. 54, Moscow, Russia \\ ${ }^{3}$ International Tomography Center SB RAS, 630090, Institutskaya str. 3A, Novosibirsk, Russia \\ ${ }^{4}$ Novosibirsk State University, 630090, Pirogova str. 2a, Novosibirsk, Russia \\ ${ }^{5}$ Institute of Chemistry of High-Purity Substances RAS, 603950, Tropinina St. 49, Nizhny Novgorod, Russia \\ mkondrin@hppi.troitsk.ru
}

PACS 33.15.Pw, 33.50.Dq, 42.50.Ex, 63.20.kp, 63.20.P

DOI 10.17586/2220-8054-2018-9-1-52-54

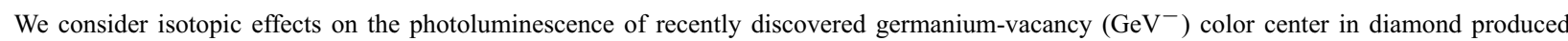
by the high-pressure high-temperature (HPHT) treatment. It was demonstrated that the influence of isotopic composition on the position of zero-phonon line (ZPL) and its first vibronic peak (local vibrational mode, LVM) provides valuable information on the electronic and structural properties of this center.
\end{abstract}

Keywords: diamond, defects, color centers, split-vacancy, high pressures.

Received: 19 June 2017. Revised: 15 December 2017

Investigation of isotopic effects on the photoluminescence of split-vacancy centers in diamond allows one to obtain deeper insight into their electronic, structural and optical properties. Due to the bright and almost monochromatic luminescence, the two already-known representatives of these class of defects $\mathrm{SiV}$ and recently discovered GeV [1-4] are considered as possible candidates for single-phonon emitters in quantum communications or photoluminescence markers in biomedical applications. Although the attractiveness of these centers is mostly caused by the large Debye-Waller factor (the intensity of the ZPL relative to the overall intensity of the center emission), the study of the luminescence sideband is still very informative.

In this paper, we discuss only negatively charged $\mathrm{GeV}$ center which was produced in nano- and microdiamonds by HPHT synthesis from organic substances $[4,5]$. This split-vacancy complex consists of impurity atoms located almost midway between two vacant cites. Ideally the symmetry of the center is $\overline{3} \mathrm{~m}$. However, due to the incompletely filled doubly degenerate impurity electron level in the diamond band gap, this structure is Jahn-Teller unstable and the degeneracy should be lifted. Thus, the actual symmetry of the center should be lower. It is believed that the physical mechanism, which lifts this degeneracy in split-vacancy centers is caused by the spinorbit coupling $[4,6,7]$. Thus, the fluorescence line caused by the promotion of an electron from the lower $e_{u}$ level to this incompletely filled $e_{g}$ one (with separation energy of about $2.059 \mathrm{eV}$ ), is split into quadruplet $\mathrm{Z}_{1-4}$ with two characteristic splitting energies 0.2 and $1.1 \mathrm{THz}$ (corresponding to the energy difference in $e_{g}$ and $e_{u}$ levels respectively). This splitting is clearly seen in Fig. 1a-b) and Fig. 2a). Still, according to electronic paramagnetic resonance measurements, the trigonal symmetry of the center is retained $[8,9]$. Oscillation of the heavy impurity Ge atom gives rise to LVM in the diamond phonon spectrum which is observed $\approx 45 \mathrm{meV}$ apart from the strongest component of ZPL line in the fluorescence sideband of this center (see Fig. 1a-b). Both the absolute energy of the strongest component of ZPL $\left(Z_{1}\right)$ and relative energy of LVM $\left(\mathrm{L}_{1}\right)$ almost linearly depends on the isotope number of Ge atom $m$ (see Fig. 1c). In the harmonic approximation, the LVM energy scales as a square root of the mass of oscillating atom but due to the small relative range $\delta m=m-m^{\prime}$ of isotope numbers of Ge atoms (70-76) it can be approximated by the linear dependence with the coefficient $\frac{\delta L_{1}}{\delta m} \approx \frac{L_{1}}{2 m}$ well reproduced in experiment (see Fig. 1c).

On the other hand, a rather strong linear dependence of absolute ZPL energy on Ge isotope number is an indication of different binding energies of the excited and ground electronic states of the GeV center (see inset in Fig 2). According to the Keil model [10] the frequency defect $\Delta$ (the difference in binding energies between the excited and the ground electronic states) leads to the following dependence of the isotopic shift of ZPL energy $(\delta Z)$ on the mass of vibrating atom: $\delta Z=\left(1-\left(\mathrm{m} / \mathrm{m}^{\prime}\right)^{1 / 2}\right) \Delta / 2$. If the isotope number difference $\delta m$ is small, then this relation can be reduced to the simple linear dependence: $\frac{\delta Z}{\delta m} \approx \frac{\Delta}{4 m}$. So the observed isotopic shift of the $\mathrm{ZPL}$ in $\mathrm{GeV}$ center indicates that the binding energy of Ge atom in the two-vacancy void of diamond lattice in 

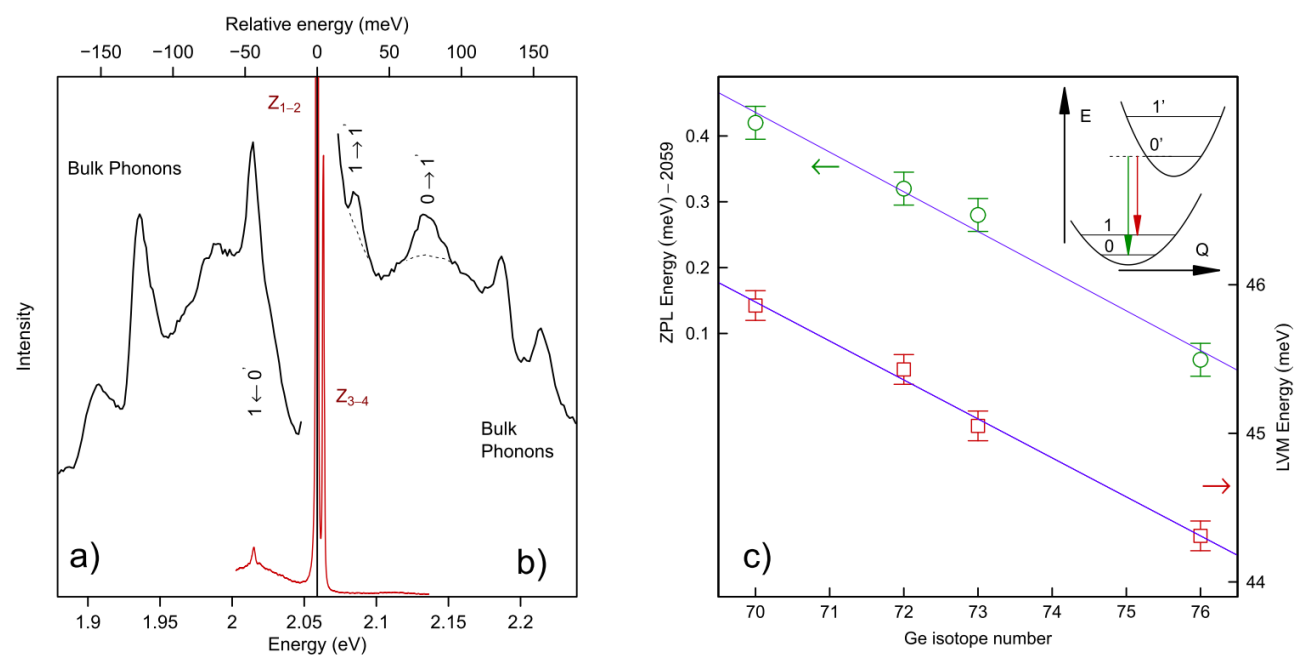

FIG. 1. Panels a) and b) represent PL and PLE sidebands respectively of $\mathrm{GeV}^{-}$center taken at $T=77 \mathrm{~K}$. The thin line in the middle of the plot represents photoluminescence of $\mathrm{GeV}^{-}$center around $\mathrm{ZPL}\left(\mathrm{Z}_{1-4}\right)$. c) the isotope shift of $\mathrm{Z}_{1} \mathrm{ZPL}$ component and LVM energy. Inset in panel c) represents the Huang-Rhys model where the arrows correspond to the energies of ZPL and LVM transitions in photoluminescence

the excited electronic state is almost $50 \%$ higher than in the ground one (about 67 and $45 \mathrm{meV}$ respectively). A similar isotopic shift was previously observed in $\mathrm{SiV}^{-}$center too and was also attributed to the difference in the potential surface curves in the excited and the ground electronic states [11].

The frequency defect $\Delta$ can be also observed in the difference between luminescence and absorption sidebands of $\mathrm{GeV}^{-}$center as shown in Fig. 1a-b. Actually, the high-energy sideband was registered using the photoluminescence excitation technique, but it can be considered as a more sensitive substitute for light absorption. The two sharp features in PLE sideband correspond to transitions $1 \rightarrow 1^{\prime}$ and $0 \rightarrow 1^{\prime}$, while in the luminescence sideband, only one line corresponding to transition $0^{\prime} \rightarrow 1$ is observed (here tick marks designate excited electronic state). This yields a slightly different curvature for the potential surface in the excited state $(73 \mathrm{meV})$ than the value obtained from isotopic shifts but it can be caused by anharmonicity effects not considered in this simple model.

The presence of an anharmonicity effects manifests itself in the isotopic shifts of ZPL caused by substitution of carbon atoms $\left({ }^{12} \mathrm{C}\right.$ to $\left.{ }^{13} \mathrm{C}\right)$. In this case, the isotopic shift reaches an impressive value of $3.2 \mathrm{meV}$. This can only be partially accounted for by isotopic contraction of diamond lattice $(\approx 0.8 \mathrm{meV}$ of this value $)$. Because carbon atoms do not directly participate in the local oscillations (it can be demonstrated by independence of LVM energy on carbon atoms mass, Fig. 2a), so the remaining part of this energy shift should be attributed to anharmonicity effects [12] or to some "silent" vibronic modes (involving oscillation of carbon atoms), which for some reason, does not contribute to the sideband of this center.

Note that the isotopic substitution of either impurity or carbon atoms both indicates that LVM is due to the vibrations of impurity atom alone. These findings might be a hint of the possible resulting symmetry (broken due to the Jahn-Teller effect) of this center.

In the previous consideration, we follow the Franck-Condon principle (also known as the Huang-Rhys model) which assumes that the electronic dipole moment of transition does not depend on the coordinates of the impurity atom. This is a natural assumption when one is dealing with allowed electronic transitions, which is the case for the $\mathrm{GeV}$ center. However, in previous studies [13,14], there is a tendency to consider the next term in the decomposition of the electronic dipole moment on the coordinates of impurity atom (the Herzberg-Teller effect) as an additional free parameter of the model that also might explain asymmetry in the luminescence and the absorption sidebands. Remarkably, the isotopic shifts also provide a means for experimental evaluation of the relative amplitude of the Franck-Condon and Herzberg-Teller effects (see Fig. 2b). In the Herzberg-Teller treatment, the change of the impurity atom mass should influence its dynamics that, in turn, should bring about relative changes of vibrational modes amplitudes on either sideband of ZPL. This is not actually observed in the substitution of Ge isotopes in $\mathrm{GeV}$ center. On the other hand, the energy shift of LVM line (caused by the Franck-Condon effect) is clearly observed in the same experiment, so this discrepancy demonstrates negligibility of the Herzberg-Teller effect in $\mathrm{GeV}^{-}$center. 

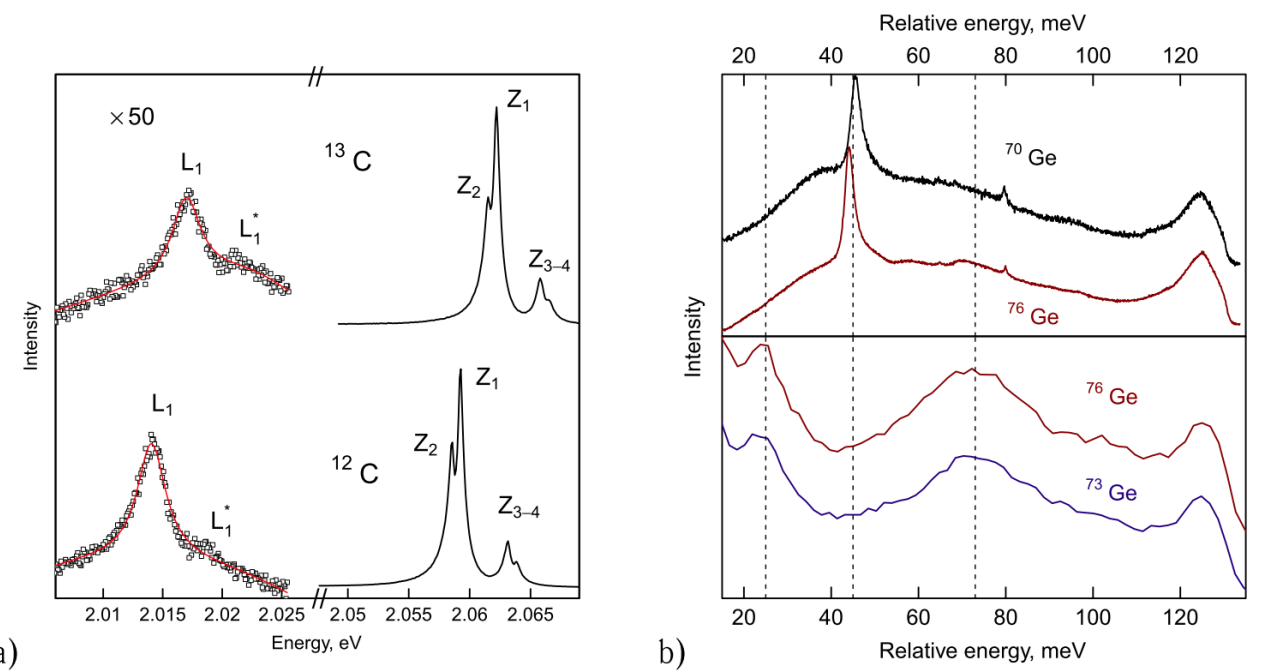

FIG. 2. a) Isotopic shift of ZPL $\left(\mathrm{Z}_{1-4}\right)$ and $\mathrm{LVM}\left(\mathrm{L}_{1}\right)$ on the isotope substitution of carbon atoms observed at $T=4.2 \mathrm{~K}$. b) Luminescence (upper part) and absorption (lower panel) sidebands relative to $\mathrm{Z}_{1}$ line of $\mathrm{GeV}^{-}$center in samples with different $\mathrm{Ge}$ isotopes $(T=77 \mathrm{~K})$

We conclude that HPHT synthesis is a powerful tool for the production of optical color centers in diamond which are in demand for potential application and (as a side effect) it is also capable of controlled introduction of the pure isotope components into resulting products. As a result, the study of isotopic effects on the properties of color centers in diamond enables one to obtain useful information regarding the electronic and structural properties of these centers.

\section{Acknowledgements}

This work was supported by the RFBR grant 17-52-50075. Photoluminescence excitation measurements were supported by FASO Russia (project 0333-2016-0001).

\section{References}

[1] Iwasaki T., Ishibashi F., Miyamoto Y., Doi Y., Kobayashi S., Miyazaki T., Tahara K., Jahnke K. D., Rogers L. J., Naydenov B., Jelezko F., Yamasaki S., Nagamachi S., Inubushi T., Mizuochi N., Hatano M. Germanium-Vacancy Single Color Centers in Diamond. Scientific Reports, 2015, 5, P. 12882.

[2] Palyanov Y. N., Kupriyanov I. N., Borzdov Y. M., Surovtsev N. V. Germanium: a new catalyst for diamond synthesis and a new optically active impurity in diamond. Scientific Reports, 2015, 5, P. 14789.

[3] Ralchenko V. G., Sedov V. S., Khomich A. A., Krivobok V. S., Nikolaev S. N., Savin S. S., Vlasov I. I., Konov V. I. Observation of the Ge-vacancy color center in microcrystalline diamond films. Bulletin of the Lebedev Physics Institute, 2015, 42, P. 165.

[4] Ekimov E. A., Lyapin S. G., Boldyrev K. N., Kondrin M. V., Khmelnitskiy R., Gavva V. A., Kotereva T. V., Popova M. N. Germaniumvacancy color center in isotopically enriched diamonds synthesized at high pressures. JETP Letters, 2015, 102 (11), P. 701-706.

[5] Ekimov E. A., Kondrin M. V. Vacancy-impurity centers in diamond: perspectives of synthesis and applications. Physics Uspekhi, 2017, 60 (6), P. 539.

[6] Rogers L. J., Jahnke K. D., Doherty M. W., Dietrich A., McGuinness L. P., Müller C., Teraji T., Sumiya H., Isoya J., Manson N. B., Jelezko F. Electronic structure of the negatively charged silicon-vacancy center in diamond. Phys. Rev. B, 2014, 89 (23), P. 235101.

[7] Gali A., Maze J. R. Ab initio study of the split silicon-vacancy defect in diamond: Electronic structure and related properties. Phys. Rev. $B, 2013, \mathbf{8 8}(23)$, P. 235205.

[8] Nadolinny V., Komarovskikh A., Palyanov Y., Kupriyanov I., Borzdov Y., Rakhmanova M., Yuryeva O., Veber S. EPR study of Si- and Ge-related defects in HPHT diamonds synthesized from Mg-based solvent-catalysts. physica status solidi (a), 2016, 213 (10), P. $2623-2628$.

[9] Komarovskikh A., Dmitriev A., Nadolinny V., Palyanov Y. A DFT calculation of EPR parameters of a germanium-vacancy defect in diamond. Diamond and Related Materials, 2017, 76, P. 86 - 89.

[10] Keil T. H. Shapes of Impurity Absorption Bands in Solids. Phys. Rev., 1965, 140 (2A), P. A601-A617.

[11] Dietrich A., Jahnke K. D., Binder J. M., Teraji T., Isoya J., Rogers L. J., Jelezko F. Isotopically varying spectral features of silicon-vacancy in diamond. New Journal of Physics, 2014, 16 (11), P. 113019.

[12] Ekimov E. A., Krivobok V. S., Lyapin S. G., Sherin P. S., Gavva V. A., Kondrin M. V. Anharmonicity effects in impurity-vacancy centers in diamond revealed by isotopic shifts and optical measurements. Phys. Rev. B, 2017, 95 (9), P. 094113.

[13] Osad'ko I. S. Determination of electron-phonon coupling from structured optical spectra of impurity centers. Soviet Physics Uspekhi, 1979, 22 (5), P. 311

[14] Naumova N., Vasil'eva I., Naumov A., Osad'ko I. Evaluation of parameters of intramolecular interaction from absorption and fluorescence spectra of substituted arylpolyene with poor resolved vibrational structure. Journal of Luminescence, 2005, 111 (1-2), P. 37 - 45. 